\title{
Beating the Stoner Criterion Using Molecular Interfaces
}

Fatma Al Ma'Mari, ${ }^{1 *}$ Timothy Moorsom, ${ }^{1 *}$ Gilberto Teobaldi, ${ }^{2}$ William Deacon, ${ }^{1}$ Thomas

Prokscha, ${ }^{3}$ Hubertus Luetkens, ${ }^{3}$ Steve Lee, ${ }^{4}$ George E. Sterbinsky, ${ }^{5}$ Dario A. Arena, ${ }^{5}$ Donald A. MacLaren, ${ }^{6}$ Machiel Flokstra,${ }^{4}$ Mannan Ali, ${ }^{1}$ May Wheeler, ${ }^{1}$ Gavin Burnell, ${ }^{1}$ Bryan J. Hickey $^{1}$ and Oscar Cespedes ${ }^{1, \dagger}$

1. School of Physics \& Astronomy, University of Leeds, LS2 9JT, United Kingdom.

2. Stephenson Institute for Renewable Energy, Department of Chemistry, University of Liverpool, L69 3BX, United Kingdom.

3. Laboratory for Muon-Spin Spectroscopy, Paul Scherrer Institut, 5232 Villigen,

Switzerland.

4. School of Physics \& Astronomy, SUPA, University of St. Andrews, KY16 9SS, United

Kingdom.

5. Photon Sciences Directorate, Brookhaven National Lab, Upton, NY, United States.

6. School of Physics \& Astronomy, SUPA, University of Glasgow, G12 8QQ, United

Kingdom.

*These authors contributed equally to this work

It is accepted that only three elements are ferromagnetic at room temperature, the transition metals iron, cobalt and nickel. The Stoner criterion explains why, for example, iron is ferromagnetic but manganese is not, even though both elements have an unfilled $3 d$ shell and are adjacent in the periodic table: the product of the density of states with the exchange integral must be greater than unity for spontaneous ordering to emerge. ${ }^{1,2}$ Here, we demonstrate that it is possible to alter the electronic states of nonferromagnetic materials, such as diamagnetic copper and paramagnetic manganese, in 
order to drive them ferromagnetic at room temperature. This remarkable effect is achieved via interfaces between metallic thin films and $C_{60}$ molecular layers. The emergent ferromagnetic state can exist over several layers of the metal before being quenched at large sample thicknesses by the material's bulk properties. While the induced magnetisation is easily measurable by magnetometry, low energy muon spin spectroscopy $^{3}$ provides insight into its magnetic distribution by studying the depolarisation process of low energy muons implanted in the sample. This technique indicates localized spin-ordered states at and close to the metallo-molecular interface. Density functional theory simulations suggest a mechanism based on magnetic hardening of the metal atoms due to electron transfer. ${ }^{4,5}$ This opens a path for the exploitation of molecular coupling to design magnetic metamaterials using abundant, non-toxic elements such as organic semiconductors. Charge transfer at molecular interfaces can then be used to control spin polarisation or magnetisation, with far reaching consequences in the design of devices for electronic, power or computing applications. ${ }^{6,7}$

Multifunctional materials with the spin degree of freedom such as multiferroics, magnetic semiconductors and molecular magnets have all aroused huge interest as potentially transformative components in quantum technologies. ${ }^{8-12}$ Strategies used to bring magnetic ordering to these materials typically rely on the inclusion of magnetic transition metals, heavy elements with a large atomic moment or rare earths. In thin film structures, proximity effects and coupling at interfaces play an essential role. ${ }^{13,14}$ This is especially the case for molecular spintronics, ${ }^{15,16}$ where organic thin films grown on copper have demonstrated spin filtering. ${ }^{17}$ The organic magnetic coupling can propagate for long distances in systems such as nanoscale vortex-like configurations or nanoskyrmion lattices. ${ }^{18}$ 
We choose $\mathrm{C}_{60}$ as a model molecule due to its structural simplicity and robustness as well as its high electron affinity. $\mathrm{C}_{60} /$ transition metal complexes exhibit strong interfacial coupling between metal $3 d_{z}$ electrons and molecular $\pi$-bonded $p$ electrons. The potential created by the mismatch of molecular and metal work functions leads to a partial filling of the interface states. ${ }^{19-21}$ Other molecules with close electron affinity and the potential for $3 d_{\mathrm{z}} / p$ coupling could be used to similar effect. In the case of $\mathrm{C}_{60}$ on metallic substrates such as $\mathrm{Cu}$ films, the charge transfer from the metal can be of up to $3 \mathrm{e}^{-}$per molecule and leads to a metallisation of the interface. ${ }^{22}$ Magnetic polarisation in fullerenes induced by spin injection or charge transfer may extend for long distances owing to low spin orbit coupling and the absence of a hyperfine interaction..$^{23,24}$

In the metal, it is expected that the charge transfer will be quickly screened by free electrons. A priori, there would be no reason to consider that a spin-unpolarized molecule would change the magnetic state of a metallic film. However, we find that the charge transfer and surface reconstruction at the interface ${ }^{25}$ can lead to an emergent magnetisation in both the metal and the molecule. Magnetometry measurements of $\mathrm{C}_{60} / \mathrm{Cu}$ and $\mathrm{C}_{60} / \mathrm{Mn}$ multilayers show hysteresis at room temperature. The magnetization disappears when all the transition metal-molecular interfaces are decoupled via an $\mathrm{Al}$ or $\mathrm{Al}_{2} \mathrm{O}_{3}$ spacer layer (Fig. 1).

Changes in the Density of States (DOS) of the metal may be larger close to the interface, but should be screened deeper within the material. If the film is thick enough, the bulk properties of the metal are expected to dominate and quench the magnetization. This effect is shown in figure 2: the magnetisation of $\mathrm{C}_{60} / \mathrm{Cu}$ and $\mathrm{C}_{60} / \mathrm{Mn}$ multilayers decays once the metallic film thickness exceeds 2-3 nm. Decreasing coupling between the top and bottom interfaces of a metal layer may also play a role.

The magnetisation of $\mathrm{C}_{60} / \mathrm{Cu}$ samples is 3-4 times stronger than for $\mathrm{C}_{60} / \mathrm{Mn}$, which is probably due to the better lattice matching and larger charge transfer between copper and 
$\mathrm{C}_{60} \cdot{ }^{19}$ However, bulk manganese is paramagnetic and much closer to complying with the Stoner criterion than diamagnetic copper due to the larger exchange interactions and density of states at the Fermi level $\left(\mathrm{DOS}\left(\mathrm{E}_{\mathrm{F}}\right)\right) .{ }^{26}$ This may be correlated with the propagation length of the effect, which persists for sample thicknesses five times longer in $\mathrm{Mn}$ than in $\mathrm{Cu}$. $\mathrm{C}_{60} / \mathrm{Mn}$ multilayers also show a larger paramagnetic slope of the magnetisation than decoupled manganese -see supplementary information (SI), Figs. S.1-S.4. Both systems exhibit anisotropy with an easy axis that lies in the plane of the film, and out-of-plane saturation fields of $\sim 10-15 \mathrm{kOe}$ at room temperature (inset in Fig. 2b). The $\mathrm{Cu}$ and $\mathrm{Mn}$ samples degrade with time, and the magnetization drops over several days or weeks depending on the layer structure and protective cap used (SI, Fig. S.5).

With a view to explore the dependence of magnetisation on interfacial coupling, we have fabricated samples with different number of $\mathrm{C}_{60}(15 \mathrm{~nm}) / \mathrm{Cu}(2.5 \mathrm{~nm})$ junctions. The magnetic moment of these multilayers is proportional to the number of $\mathrm{Cu}-\mathrm{C}_{60}$ interfaces, suggesting that the magnetism is indeed due to molecular coupling (Fig. 2a inset). However, the amount of $\mathrm{Cu}$ and $\mathrm{C}_{60}$ is also increased as we grow more layers. In order to ensure that the magnetisation is not simply proportional to the amount of material deposited, we perform a related set of measurements where the total sample thickness is kept constant: $9 \mathrm{~nm}$ of Cu and $81 \mathrm{~nm}$ of $\mathrm{C}_{60}$, but split into different number of $\mathrm{C}_{60} / \mathrm{Cu}$ repeats (SI, Fig. S.6). There, the magnetism also increases with the number of interfaces; e.g. magnetic moment of $\left[\mathrm{C}_{60}(16.2\right.$ $\mathrm{nm}) / \mathrm{Cu}(1.8 \mathrm{~nm})] \times 5>$ moment of $\left[\mathrm{C}_{60}(27 \mathrm{~nm}) / \mathrm{Cu}(3 \mathrm{~nm})\right] \times 3$. However, trying to split the sample into $\mathrm{Cu}$ films of $\sim 1.5 \mathrm{~nm}$ or thinner results in discontinuous layers and a drop in the magnetisation. This thickness and interface dependence of the magnetisation could not arise from contaminants, and X-ray spectroscopy did not show the presence of impurities (SI, Figs. S.7-S.11). 
Magnetometry measurements show that the magnetisation is dependent on the thickness of the metal but not on that of the molecular film as long as the latter is continuous and smooth $(\sim 10-20 \mathrm{~nm})$. However, magnetometry by itself cannot determine where the magnetisation is located or how much of it corresponds to each material. Conversely, low energy muon spin rotation $(\mu \mathrm{SR})$ provides a magnetic profile of the sample ${ }^{27}$ and has been applied successfully to other metallo-molecular systems. ${ }^{3}$ Here, a beam of almost fully polarised positive muons $\left(\mu^{+}\right)$is moderated to $\mathrm{keV}$ energies so that their tunable stopping range is tens to hundreds of $\mathrm{nm}$. The local polarisation at the $\mu^{+}$stopping depth is probed through the detection of decay positrons, preferentially emitted along the muons' spin direction.

We use this technique to study two samples: $\mathrm{Cu}-\mathrm{C}_{60}$ is a magnetic multilayer with the structure (from the bottom): $\mathrm{Ta}(5) / \mathrm{C}_{60}(20) / \mathrm{Cu}(2.5) / \mathrm{C}_{60}(50) / \mathrm{Au}(10)$-thickness in brackets in nm. $\mathrm{Cu}-\mathrm{Al}_{2} \mathrm{O}_{3}-\mathrm{C}_{60}$ is a decoupled, diamagnetic reference multilayer with $\mathrm{Al}_{2} \mathrm{O}_{3}$ layers in between the $\mathrm{Cu}$ and $\mathrm{C}_{60}$ (Fig. 3a). The uppermost gold film slows injected $\mu^{+}$and protects the inner layers from oxidation. The total sample structure is designed to allow the active layers to be probed with a range of accessible $\mu^{+}$energies and to maximise the stopping profile at the regions of interest, i.e. close to the $\mathrm{Cu}-\mathrm{C}_{60}$ interface. The $\mathrm{Cu}$ thickness is chosen to obtain the highest magnetisation (Fig. 2a). Muon stopping profiles and further experimental details can be found in the SI, section $\S$ S.3 and Figs. S.12-S.13.

Muons with $4 \mathrm{keV}$ implantation energy probe the identical uppermost $\mathrm{C}_{60}(50) / \mathrm{Au}(10)$ layers of both samples. Nevertheless, the zero field $\mu \mathrm{SR}$ measurements at $250 \mathrm{~K}$ demonstrate a significant difference: for the magnetic $\mathrm{Cu}-\mathrm{C}_{60}$ sample a fraction of $\approx 10 \%$ of the muon spin polarization is rapidly lost -indicating that about $10 \%$ of the region sampled by the muons is affected by the magnetism (Fig. 3b). This points to additional sources of magnetic flux in the $\mathrm{Cu}-\mathrm{C}_{60}$ sample. In this region, the additional flux most likely arises from stray dipolar fields, 
since at low temperatures $(20 \mathrm{~K})$ we find that $75 \%$ of the $\mu^{+}$implanted in the $\mathrm{C}_{60}$ layer form a bound electron-muon state called muonium which can only be observed in a non-magnetic environment. ${ }^{28,29}$ This strongly indicates that the $\mathrm{C}_{60}$ layer is for the most part free of magnetic moments, and suggests that the magnetism is localized at the $\mathrm{Cu}-\mathrm{C}_{60}$ interface. Due to the presence of muonium in $\mathrm{C}_{60}$ the data analysis is difficult, but further support for this scenario comes from the energy/depth dependence of the $\mu \mathrm{SR}$ data at low and high temperatures. At $20 \mathrm{~K}$ the observable muonium fraction decreases in the magnetic $\mathrm{Cu}-\mathrm{C}_{60}$ sample as one approaches the $\mathrm{Cu}$ layer (SI, Fig. S.14). Analogously, at $250 \mathrm{~K}$ the difference between the spectra of the two samples increases for the $6 \mathrm{keV}$ data and is even greater at 8 $\mathrm{keV}$, the energy at which the muons most heavily sample the $\mathrm{Cu}$ layer (Fig. 3b). If the $\mathrm{Cu}$ were non-magnetic, one would expect an overall increase of the muon polarization, which is obviously not observed.

Another means of locating the magnetism in the $\mathrm{Cu}-\mathrm{C}_{60}$ sample is to study its $\mu \mathrm{SR}$ response in the zero field remanent state. Both samples contain an oscillation at $0.4 \mathrm{MHz}$ due to muonium formed in semiconducting $\mathrm{C}_{60}$. After applying an external field of $300 \mathrm{Oe}$, this signal is shifted to $0.6 \mathrm{MHz}$, which we attribute to a small residual field of approximately 0.3 Oe in the apparatus. Nonetheless, the remanent $8 \mathrm{keV} \mu \mathrm{SR}$ spectra shown in Fig. $3 \mathrm{~b}$ are clearly different from the virgin spectra for the magnetic $\mathrm{Cu}-\mathrm{C}_{60}$ sample, while only subtle changes can be observed for the non-magnetic reference sample $\mathrm{Cu}-\mathrm{Al}_{2} \mathrm{O}_{3}-\mathrm{C}_{60}$. The striking new feature related to the remanent state of $\mathrm{Cu}-\mathrm{C}_{60}$ is an additional oscillation at $\approx 0.75 \mathrm{MHz}$ which is not observed for the non-magnetic $\mathrm{Cu}-\mathrm{Al}_{2} \mathrm{O}_{3}-\mathrm{C}_{60}$ (Fig. 3c). This new frequency can be naturally explained by an additional magnetic field of 0.1 Oe at the muonium site in close proximity to the $\mathrm{Cu}$ layer. The amplitude of this signal follows the fraction of $\mu^{+}$stopped in the $\mathrm{Cu}$ layer (Fig. 3d), while the non-magnetic signal at $0.6 \mathrm{MHz}$ is anti-correlated to this 
fraction. Altogether, the low energy $\mu \mathrm{SR}$ data fully support the notion of a magnetic moment being localized in the metallic layer and the immediate $\mathrm{Cu}-\mathrm{C}_{60}$ interface.

To search for the origins of the induced magnetisation, we have modelled the $\mathrm{Cu}-\mathrm{C}_{60}$ interface via Density Functional Theory (DFT; SI Section $\S S .4)$. The molecular roughness of the $\mathrm{C}_{60}$ films has been accounted for via several interface models based on: $i$. the single crystal 7-vacancy $\mathrm{Cu}(111)$ reconstruction, ${ }^{20}{ }^{2 i}$. $\mathrm{C}_{60}$ encapsulation into adsorbed $\mathrm{Cu}(111)$ films $\left(\mathrm{C}_{60} @ \mathrm{Cu}\right)$, and iii. $\mathrm{Cu}(111)$ growth into the pits of the $\mathrm{C}_{60}$-film $\left(\mathrm{Cu} @ \mathrm{C}_{60}\right)$, see Fig. 4a. Regardless of the adopted DFT flavour (SI, Figs. S.15-S.19), we find a non-magnetic ground state for all the considered interfaces. With the exception of the thicker $\mathrm{C}_{60} /$ slab interfaces and $\mathrm{C}_{60} @ \mathrm{Cu}$ models prepared with short (1.5-2 $\AA$ ) initial $\mathrm{Cu}-\mathrm{C}$ distances, all the models exhibit a convex (positive) curvature of $\operatorname{DOS}\left(\mathrm{E}_{\mathrm{F}}\right)$-see figure $4 \mathrm{~b}$. Within the mean-field itinerant electron model (SI, Fig. S.20), convex $\mathrm{DOS}\left(\mathrm{E}_{\mathrm{F}}\right)$ can lead, for sufficiently high external magnetic fields, to a spontaneous first-order para to ferromagnetic metamagnetic transition. For the computed $\mathrm{DOS}\left(\mathrm{E}_{\mathrm{F}}\right)$ of the $\mathrm{Cu}-\mathrm{C}_{60}$ interface models, the critical field $\left(\mathrm{H}_{\mathrm{c}}\right)$ for the metamagnetic transition sharply decreases with increasing values of the Stoner exchange $\left(\mathrm{I}_{\mathrm{S}}\right)$ as $\left[1-\mathrm{I}_{\mathrm{S}} \mathrm{DOS}\left(\mathrm{E}_{\mathrm{F}}\right)\right]^{3 / 2}$.

Atom-resolved analysis of the $\mathrm{I}_{\mathrm{s}}$ parameter reveals up to a factor 4 change of the exchange strength at the $\mathrm{Cu}-\mathrm{C}_{60}$ interfaces (Fig. 4c). Magnetic hardening up to a factor of three has been previously reported for magnetic cobalt atoms contacted to $\pi$-conjugated molecules. ${ }^{5}$ The computed values of $\mathrm{I}_{\mathrm{s}}$ (from $0.86 \mathrm{eV}$ for bulk $\mathrm{Cu}$ to up to $>2.5 \mathrm{eV}$ for interfacial $\mathrm{Cu}$ atoms) suggest viable para to ferro metamagnetic transitions for fields lower than $1 \mathrm{kOe}$ for thin $\mathrm{Cu}$ layers (Fig. 4d). On this basis, we may attribute the measured ferromagnetism to a transition of the $\mathrm{Cu}-\mathrm{C}_{60}$ system in magnetic fields of 0.3-5 kOe generated during sample deposition and preparation (see Methods). Our DFT calculations predict that 77 to $95 \%$ of the 
magnetisation in the $\mathrm{Cu}-\mathrm{C}_{60}$ system will then be distributed in the metal (SI, Table S.8 and Figs. S.21-S.26), in good agreement with the muon spectroscopy data.

Although the substantial electron transfer from the Cu-layers to $\mathrm{C}_{60}\left(\geq 1.6 \mathrm{e}^{-} / \mathrm{C}_{60}\right.$ depending on the model; SI, Table S.7) is effective in altering the curvature of DOS( $\left.\mathrm{E}_{\mathrm{F}}\right)$ and increasing $\mathrm{I}_{\mathrm{s}}$, the calculated $\operatorname{DOS}\left(\mathrm{E}_{\mathrm{F}}\right) \times \mathrm{I}_{\mathrm{s}}$ product remains less than unity (SI, Table S.6), leaving the Stoner criterion unfulfilled. However, magnetometry and muon spectroscopy provide conclusive evidence for the emergence of magnetism at $\mathrm{Cu} / \mathrm{C}_{60}$ interfaces. This is probably correlated with a sharp decrease of five orders of magnitude in the ferrometamagnetic critical field made possible by $\mathrm{C}_{60}$-induced magnetic hardening of $\mathrm{Cu}$. Similar effects due to charge transfer could also take place in other hybrid metallo-organic ${ }^{17}$ and $d^{0}$ magnetic systems. ${ }^{30}$ In order to maximise the potential of this effect, it should be possible to look for molecules with large electron affinity such as polyoxometalates and metals with a large exchange integral such as zinc. However, good band and structural matching will be needed to obtain significant results. Manipulating the charge transfer by applying electric potentials or through energy band matching could lead to applications in molecular memories or devices such as spin capacitors.

1 Stoner, E. C. Collective electron ferromagnetism. Proceedings of the Royal Society of London Series a-Mathematical and Physical Sciences 165, 0372-0414 (1938).

2 Stoner, E. C. Collective electron ferromagnetism II. Energy and specific heat. Proceedings of the Royal Society of London Series a-Mathematical and Physical Sciences 169, 0339-0371 (1939).

3 Drew, A. J. et al. Direct measurement of the electronic spin diffusion length in a fully functional organic spin valve by low-energy muon spin rotation. Nature Materials 8, 109-114 (2009).

$4 \quad$ Vandewal, K. et al. Efficient charge generation by relaxed charge-transfer states at organic interfaces. Nature Materials 13, 63-68 (2014).

5 Callsen, M., Caciuc, V., Kiselev, N., Atodiresei, N. \& Bluegel, S. Magnetic Hardening Induced by Nonmagnetic Organic Molecules. Physical Review Letters 111, 106805 (2013).

6 Moodera, J. S., Koopmans, B. \& Oppeneer, P. M. On the path toward organic spintronics. MRS Bulletin 39, 578-581 (2014), and references therein.

7 Raman, K. V. Interface-assisted molecular spintronics. Applied Physics Reviews 1, 031101(2014).

8 Beeler, M. C. et al. The spin Hall effect in a quantum gas. Nature 498, 201-204 (2013).

9 Eerenstein, W., Mathur, N. D. \& Scott, J. F. Multiferroic and magnetoelectric materials. Nature 442, 759-765 (2006). 
Powell, A. K. MOLECULAR MAGNETISM A bridge to higher ground. Nature Chemistry 2, 351352 (2010).

11 Geng, Y. et al. Direct visualization of magnetoelectric domains. Nature Materials 13, $163-167$ (2014).

12 Warner, M. et al. Potential for spin-based information processing in a thin-film molecular semiconductor. Nature 503, 504-508 (2013).

13 Maccherozzi, F. et al. Evidence for a Magnetic Proximity Effect up to Room Temperature at Fe/(Ga,Mn)As Interfaces. Physical Review Letters 101, 267201 (2008).

14 Vobornik, l. et al. Magnetic Proximity Effect as a Pathway to Spintronic Applications of Topological Insulators. Nano Letters 11, 4079-4082 (2011).

15 Barraud, C. et al. Unravelling the role of the interface for spin injection into organic semiconductors. Nature Physics 6, 615-620 (2010).

16 Sanvito, S. MOLECULAR SPINTRONICS The rise of spinterface science. Nature Physics 6, 562564 (2010).

17 Raman, K. V. et al. Interface-engineered templates for molecular spin memory devices. Nature 493, 509-513 (2013).

18 Brede, J. et al. Long-range magnetic coupling between nanoscale organic-metal hybrids mediated by a nanoskyrmion lattice. Nature Nanotechnology 9, 1018-1023 (2014).

19 Pai, W. W. et al. Optimal Electron Doping of a C60 Monolayer on Cu(111) via Interface Reconstruction. Physical Review Letters 104, 036103 (2010).

$20 \mathrm{Xu}, \mathrm{G}$. et al. Detailed low-energy electron diffraction analysis of the $(4 \times 4)$ surface structure of C-60 on Cu(111): Seven-atom-vacancy reconstruction. Physical Review B 86, 075419 (2012).

21 Tamai, A. et al. Electronic structure at the $\mathrm{C}_{60} /$ metal interface: An angle-resolved photoemission and first-principles study. Physical Review B 77,075134 (2008).

22 Cho, S. W. et al. Origin of charge transfer complex resulting in Ohmic contact at the $\mathrm{C}_{60} / \mathrm{Cu}$ interface. Synthetic Metals 157, 160-164 (2007).

23 Zhang, X. et al. Observation of a large spin-dependent transport length in organic spin valves at room temperature. Nature Communications 4, 1392 doi:10.1038/ncomms2423 (2013).

24 Moorsom, T. et al. Spin-polarized electron transfer in ferromagnet/C60 interfaces. Physical Review B 90, 125311 (2014).

25 Tseng, T.-C. et al. Charge-transfer-induced structural rearrangements at both sides of organic/metal interfaces. Nature Chemistry 2, 374-379 (2010).

26 Janak, J. F. UNIFORM SUSCEPTIBILITIES OF METALLIC ELEMENTS. Physical Review B 16, 255262 (1977).

27 Morenzoni, E. et al. Implantation studies of keV positive muons in thin metallic layers. Nuclear Instruments \& Methods in Physics Research Section B-Beam Interactions with Materials and Atoms 192, 254-266 (2002).

28 Ansaldo, E. J., Niedermayer, C. \& Stronach, C. E. MUONIUM IN FULLERITE. Nature 353, 121121 (1991).

29 Duty, T. L. et al. ZERO-FIELD MU-SR IN CRYSTALLINE C-60. Hyperfine Interactions 86, 789-795 (1994).

30 Coey, J. M. D. d(0) ferromagnetism. Solid State Sciences 7, 660-667 (2005). 


\section{Figure legends}

Figure 1: Effect of molecular interfaces. Schematics and room temperature magnetisation for a $\mathrm{Ta}(5) /\left[\mathrm{C}_{60}(15) / \mathrm{Cu}(2)\right] \times 5 / \mathrm{Al}(5)$ and a $\mathrm{Ta}(5) /\left[\mathrm{C}_{60}(15) / \mathrm{Al}(3) / \mathrm{Cu}(2) / \mathrm{Al}(3)\right] \times 5$ samples -film thickness is in brackets in $\mathrm{nm}$. The $\mathrm{Cu}$-to- $\mathrm{C}_{60}$ charge transfer and interface reconstruction results in significant changes in the DOS of the metallic film and a band splitting that leads to magnetic ordering. On the other hand, an Al spacer between both materials screens the charge transfer from the $3 d$ transition metal and stops the band splitting.

Figure 2: Room temperature magnetisation for $\mathrm{Cu}$ and $\mathrm{Mn}$ films. Dashed lines are exponentially modified Gaussian fits. Error bars in thickness constitute the film roughness and in magnetisation they are calculated as the error of the mean. a. Dependence of the magnetisation with the $\mathrm{Cu}$ film thickness for a total of 145 samples with the structure $\mathrm{Ta}(5) /\left[\mathrm{C}_{60}(10-20) / \mathrm{Cu}(\boldsymbol{t}) / \mathrm{C}_{60}(10-20)\right] \times 1-5 / \mathrm{Al}(5)$. Films with $\mathrm{t} \lesssim 1-1.5 \mathrm{~nm}$ are discontinuous. Inset: Magnetic moment vs. number of $\mathrm{C}_{60}(15) / \mathrm{Cu}(2.5)$ interfaces -the moment is roughly proportional to the number of interfaces. b. As a. for Mn, with 96 samples measured. The magnetisation in $\mathrm{Mn}$ films is smaller than for $\mathrm{Cu}$, but propagates for a longer distance. Inset: Out-of-plane and in-plane magnetisation measurements of a $\left[\mathrm{C}_{60}(15) / \mathrm{Mn}(2.5)\right] \mathrm{x} 4$ sample.

Figure 3: Muon spin spectroscopy $(\mu \mathrm{SR})$ at $250 \mathrm{~K}$. Error bars are the standard error in $\sim 10^{6}$ events. a. Schematic of the experiment and samples measured: $\mathrm{Cu}-\mathrm{C}_{60}$ : $\left[\mathrm{Ta}(5) / \mathrm{C}_{60}(20) / \mathrm{Cu}(2.5) / \mathrm{C}_{60}(50) / \mathrm{Au}(10)\right] ;$ and the control $\mathrm{Cu}-\mathrm{Al}_{2} \mathrm{O}_{3}-\mathrm{C}_{60}$ : $\left[\mathrm{Ta}(5) / \mathrm{C}_{60}(20) / \mathrm{Al}_{2} \mathrm{O}_{3}(4) / \mathrm{Cu}(2.5) / \mathrm{Al}_{2} \mathrm{O}_{3}(4) / \mathrm{C}_{60}(50) / \mathrm{Au}(10)\right]$. Far-right: raw hysteresis loops. b. Zero field $\mu$ SR spectra for 4, 6, 8 and $16 \mathrm{keV}$ muon implantation energies. Tables show the fraction of muons stopped in each layer/sample. At $8 \mathrm{keV}$, zero field spectra are shown before (closed symbols) and after an applied magnetic field of 300 Oe (remanent state - open symbols), evidencing clear differences in the $\mathrm{Cu}-\mathrm{C}_{60}$ sample. c. Oscillation frequencies from fits to the data plotted in $\mathbf{b}$. Only in the $\mathrm{Cu}-\mathrm{C}_{60}$ sample, a new signal at $\sim 0.75 \mathrm{MHz}$ attributed 
to the emergent magnetisation is observed in remanence. $\mathbf{d}$. The polarisation amplitude of the magnetic remanent signal at $0.75 \mathrm{MHz}$ tracks the fraction of muons stopped in the $\mathrm{Cu}$ (maximum at $8 \mathrm{keV}$ ), whereas the signal associated to muonium at $0.6 \mathrm{MHz}$ is anti-correlated to it.

Figure 4: DFT simulations and metamagnetic modellling. a. Schematic of the molecularly rough $\mathrm{Cu}-\mathrm{C}_{60}$ interface with: atomically flat $\mathrm{C}_{60}-\mathrm{Cu}$ contacts (green square), $\mathrm{C}_{60}$ inclusion in $\mathrm{Cu}$-film $\left(\mathrm{C}_{60} @ \mathrm{Cu}\right.$; red square) and $\mathrm{Cu}$-inclusion in pits of the $\mathrm{C}_{60}$-film $\left(\mathrm{Cu} @ \mathrm{C}_{60}\right.$; black square). The $\mathrm{C}_{60} @ \mathrm{Cu}-1.5(55 \mathrm{v})$ model includes $55 \mathrm{Cu}$ vacancies. The optimised atomic structures are reported and labelled in SI, Figs. S.15-S.19. b. Total DOS around the Fermi energy $\left(\mathrm{E}_{\mathrm{F}}\right)$ for bulk-Cu and the $\mathrm{Cu}-\mathrm{C}_{60}$ interface models. c. Atom-resolved analysis of the Stoner exchange integral for the $\mathrm{Cu}$-atoms as a function of the shortest $\mathrm{Cu}-\mathrm{C}_{60}$ distance. $\mathbf{d}$. The critical magnetic field $\left(\mathrm{H}_{\mathrm{c}}\right)$ for spontanous ferromagnet metamagnetic transition as a function of Is for the models of computed positive $\operatorname{DOS}\left(\mathrm{E}_{\mathrm{F}}\right)$ curvature in $(\mathrm{b})$. The horizontal black line marks a typical magnetic field during sample preparation. Shaded area are possible values for $\mathrm{I}_{\mathrm{s}}$ in the different geometries. 
Methods Magnetic measurements were taken using a superconducting quantum interference device operated as a vibrating sample magnetometer (SQUID-VSM or SVSM) model MPMS3 from Quantum Design with resolution better than $10^{-8} \mathrm{emu}$. The thin films were deposited on $0.5 \mathrm{~mm}$ thick $\mathrm{Si} / \mathrm{SiO}_{2}$ substrates. Metals were deposited by DC magnetron sputtering at a pressure of approx. $2.5 \mathrm{mbar}\left(24 \mathrm{sccm}\right.$ of $\mathrm{Ar} ; 10^{-8} \mathrm{mbar}$ base pressure) with a deposition rate of 1-3 $\AA /$ s. $\mathrm{C}_{60}$ films were deposited by thermal evaporation from a sublimed, $99.9 \%$ purity source in an alumina boat in the same chamber at $\sim 10^{-8}$ mbar and with deposition rates of $0.5-1 \AA / \mathrm{s}$. Alumina films were grown via plasma oxidation of Al films: $\mathrm{O}_{2}$ flow of $76 \mathrm{SCCM}, 35 \mathrm{~mA}$ current. Oxygen is highly detrimental to the emergent magnetism, and samples grown in a poor vacuum $\left(\mathrm{P}_{\text {total }} \geq 2 \times 10^{-8}\right.$ or $\mathrm{P}_{\mathrm{O} 2} \geq 5 \times 10^{-10}$ mbar $)$ show no magnetisation. Ta seed layers are used to improve the sample roughness. Our thermally sublimated $\mathrm{C}_{60}$ films are relatively rough when compared to sputtered metallic films $(\sim 1 \mathrm{~nm}$ RMS roughness for $\mathrm{C}_{60} \mathrm{vs} .<0.5 \mathrm{~nm}$ in metals). The metallic films are continuous and there is no significant diffusion into the molecular film as seen in low angle X-rays (SI, Fig. S.3). Cross-sections of representative samples were analysed by transmission electron microscopy and showed the metallic layers to be continuous and the $\mathrm{C}_{60}$ layers to be polycrystalline (Fig. S.27). The films experience a magnetic field of $\sim 0.3 \mathrm{kOe}$ during growth due to an in-plane magnet and the field from the magnetron gun. They are also subject to fields of $\sim 1-5 \mathrm{kOe}$ during loading and centring in the SVSM - needed to position the sample with respect to the SQUID sensor.

Low energy muon spin spectroscopy ${ }^{31}$ utilizes positive muons to provide a probe of local magnetisation. Positive muons $\left(\mu^{+}\right)$are implanted into a sample and decay into a detectable positron and a neutrino/anti-neutrino pair. Due to charge parity violation, there is a preferred direction of emission of the positrons along the muons' spin vector. Determining the direction of the positron decay allows the precession of the muon spin to be determined and, therefore, 
the local field at the muon implantation site. A polarized high intensity beam of energetic $(\mathrm{MeV})$ positive muons ${ }^{32}$ is obtained from the decay of $\pi^{+}$, generated by a proton beam impinging on a graphite target. After moderation in a cryogenic solid Ar moderator where the beam polarisation is conserved ${ }^{33}$ the anti-muons are re-accelerated electrostatically to $\mathrm{keV}$ energies and transported by electrostatic elements to the sample. Positrons emitted from muon decay are detected by two plastic scintillator rings and the difference between the flux observed at these two detectors is used to determine the instantaneous spin direction of implanted positive muons as a function of time. The muon asymmetry data plot is then calculated as: $\mathrm{A}(\mathrm{t})=[\mathrm{NL}(\mathrm{t})-\mathrm{NR}(\mathrm{t})] /[\mathrm{NL}(\mathrm{t})+\mathrm{NR}(\mathrm{t})]$, where $\mathrm{NL} / \mathrm{R}(\mathrm{t})$ are the background corrected decay histograms of the left and right positron detector, respectively. There, the error of each bin count $n$ is given by the standard deviation of $n$. The errors of each bin in $\mathrm{A}(\mathrm{t})$ are then calculated by standard error propagation.

Standard, fixed spin-moment ${ }^{34}$ and non-collinear van der Waals (vdW) corrected Density Functional Theory (DFT) simulations were executed via the Projected Augmented Wave (PAW) method as implemented in the VASP program. ${ }^{35}$ We used the PBE exchangecorrelation (XC) functional, ${ }^{36}$ a $400 \mathrm{eV}$ plane-wave energy cut-off, $(0.2 \mathrm{eV}, 1$ st order) Methfessel-Paxton electronic smearing, ${ }^{37}$ and a 10 symmetry irreducible k-point grid for the $\mathrm{C}_{60} / 7 \mathrm{vac} \mathrm{Cu}(111)-4 \mathrm{x} 4$ models. For $\mathrm{C}_{60} @ \mathrm{Cu}(111)-8 \mathrm{x} 8$ and $\mathrm{Cu}(111)-8 \mathrm{x} 8 @ \mathrm{C}_{60}$ models only one k-point was used. The adopted atomic-force threshold for geometry optimization was $0.02 \mathrm{eV} \AA-1$. For the $7 \mathrm{vac}$ model we relaxed the topmost $5 \mathrm{Cu}$-layers together with the all the $\mathrm{C}_{60}$ atoms. All the atoms of the $\mathrm{C}_{60} @ \mathrm{Cu}(111)-8 \mathrm{x} 8$ and $\mathrm{Cu}(111)-8 \mathrm{x} 8 @ \mathrm{C}_{60}$ models were relaxed. In all cases, a vacuum separation of at least $12 \AA$ was present between replicated images of the interface models.

31 Bakule, P. \& Morenzoni, E. Generation and applications of slow polarized muons. Contemporary Physics 45, 203-225 (2004). 
32 Prokscha, T. et al. The new mu E4 beam at PSI: A hybrid-type large acceptance channel for the generation of a high intensity surface-muon beam. Nuclear Instruments \& Methods in Physics Research Section a-Accelerators Spectrometers Detectors and Associated Equipment 595, 317-331 (2008).

33 Morenzoni, E. et al. GENERATION OF VERY SLOW POLARIZED POSITIVE MUONS. Physical Review Letters 72, 2793-2796 (1994).

34 Schwarz, K. \& Mohn, P. ITINERANT METAMAGNETISM IN YCO2. Journal of Physics F-Metal Physics 14, L129-L134 (1984).

35 Kresse, G. \& Furthmuller, J. Efficient iterative schemes for ab initio total-energy calculations using a plane-wave basis set. Physical Review B 54, 11169-11186 (1996).

36 Perdew, J. P., Burke, K. \& Ernzerhof, M. Generalized gradient approximation made simple. Physical Review Letters 77, 3865-3868 (1996).

37 Methfessel, M. \& Paxton, A. T. HIGH-PRECISION SAMPLING FOR BRILLOUIN-ZONE INTEGRATION IN METALS. Physical Review B 40, 3616-3621 (1989).

Supplementary Information is linked to the online version of the paper at www.nature.com/nature.

Acknowledgments Engineering and Physical Sciences Research Council for support through grants EP/K00512X/1, EP/K036408/1, EP/J01060X/1 and EP/I004483/1. Use of the N8 (EPSRC EP/K000225/1), ARCHER (via the UKCP Consortium, EP/K013610/1), and HPC Wales High Performance Computing facilities is gratefully acknowledged. Use of the National Synchrotron Light Source, Brookhaven National Laboratory, was supported by the U.S. Department of Energy, Office of Science, Office of Basic Energy Sciences, under Contract No. DE-AC02-98CH10886.

Author Contributions F.A.M. and T.M. grew and characterized samples, conducted magnetometry and $\mu \mathrm{SR}$, and contributed to the data analysis; G.T. performed and analysed the DFT simulations; W.D. grew and characterized $\mathrm{Cu}-\mathrm{C}_{60}$ multilayers; T.P., H.L., S.L. and M.F. contributed to the design, measurement and analysis of $\mu$ SR experiments; D.A.M. contributed with TEM images and structural analysis; G.E.S. and D.A.A. performed the XMCD and XAS measurements; M.A., M.W., G.B. and B.H. contributed to the sample structure and measurement setup; OC designed the study, analysed data and wrote the manuscript. All authors discussed the results and commented on the manuscript. 
Author Information Reprints and permissions information is available at www.nature.com/reprints. The authors declare no competing financial interests. Correspondence and requests for materials should be addressed to O.C. (o.cespedes@leeds.ac.uk). 


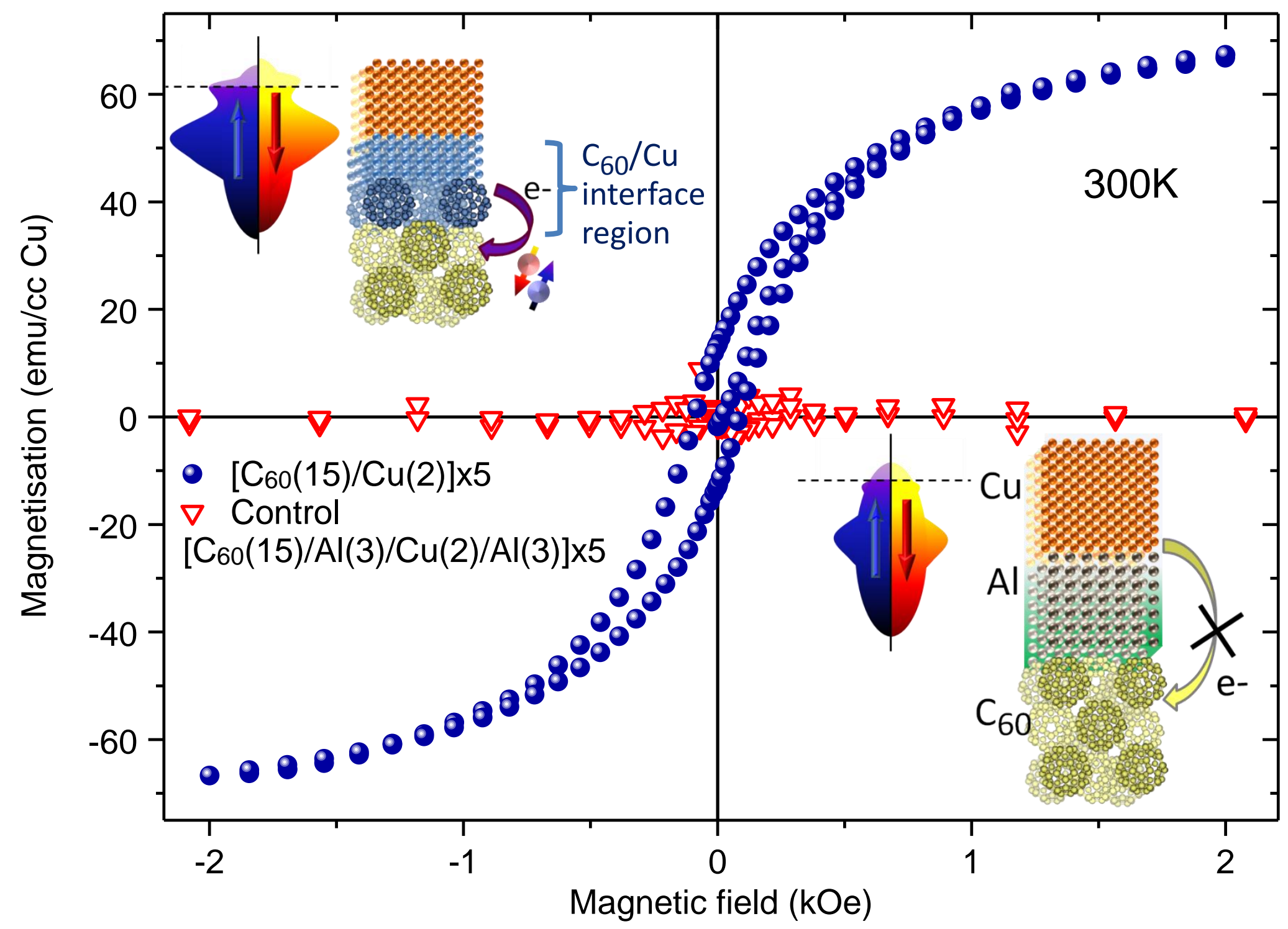



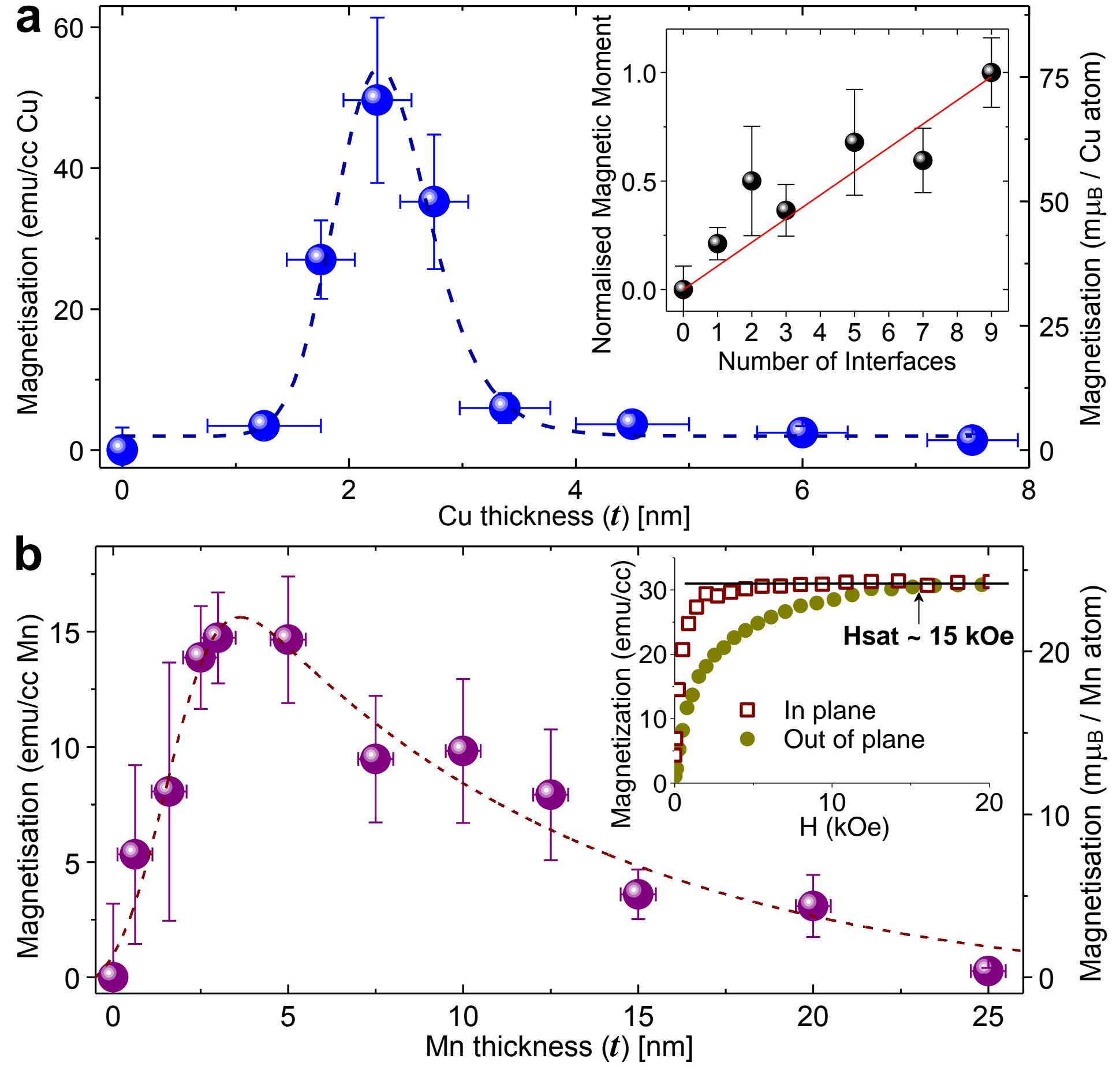

2 2 - 00000000

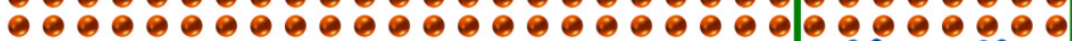

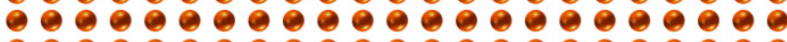

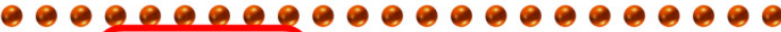
$20 \%$ -

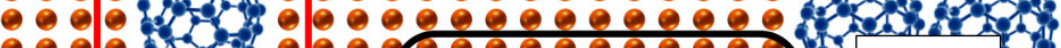

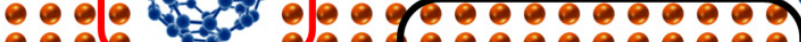

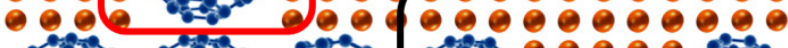

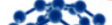
30

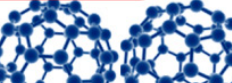

20

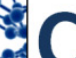

웅 (1) -
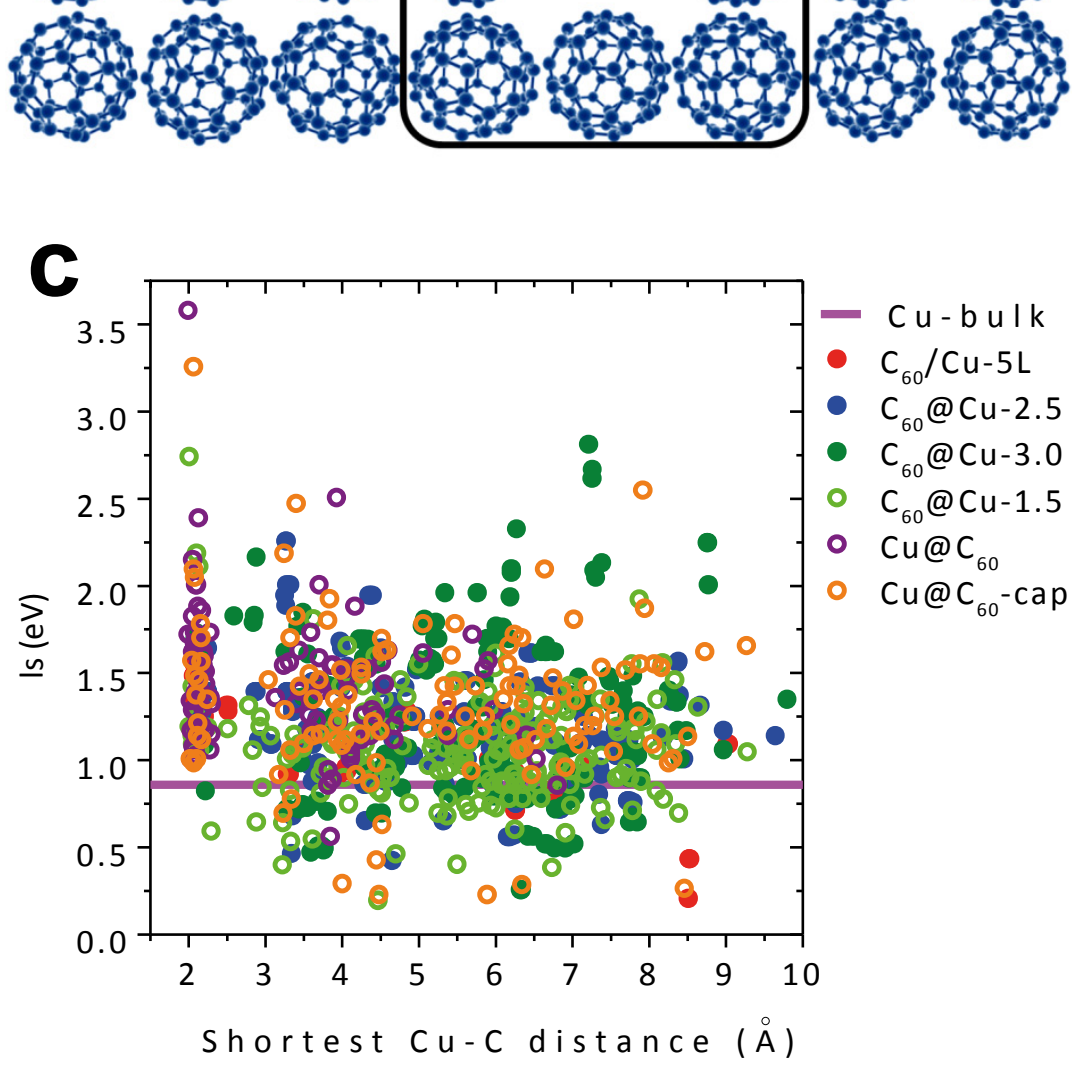

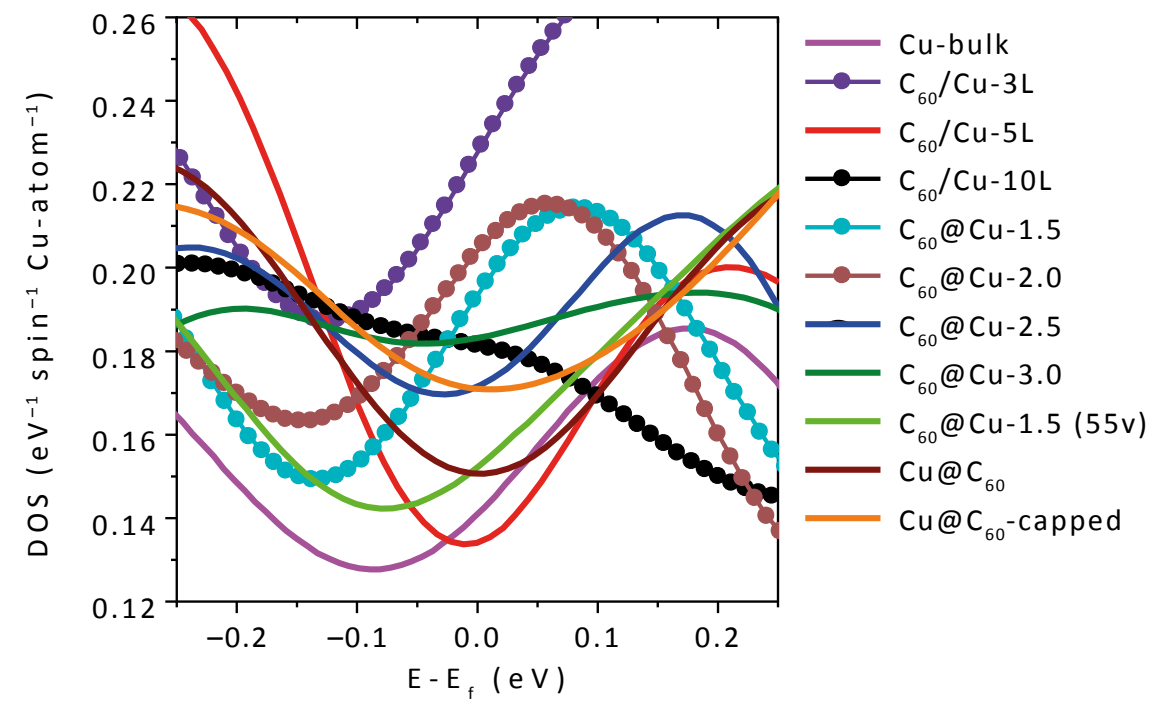

d

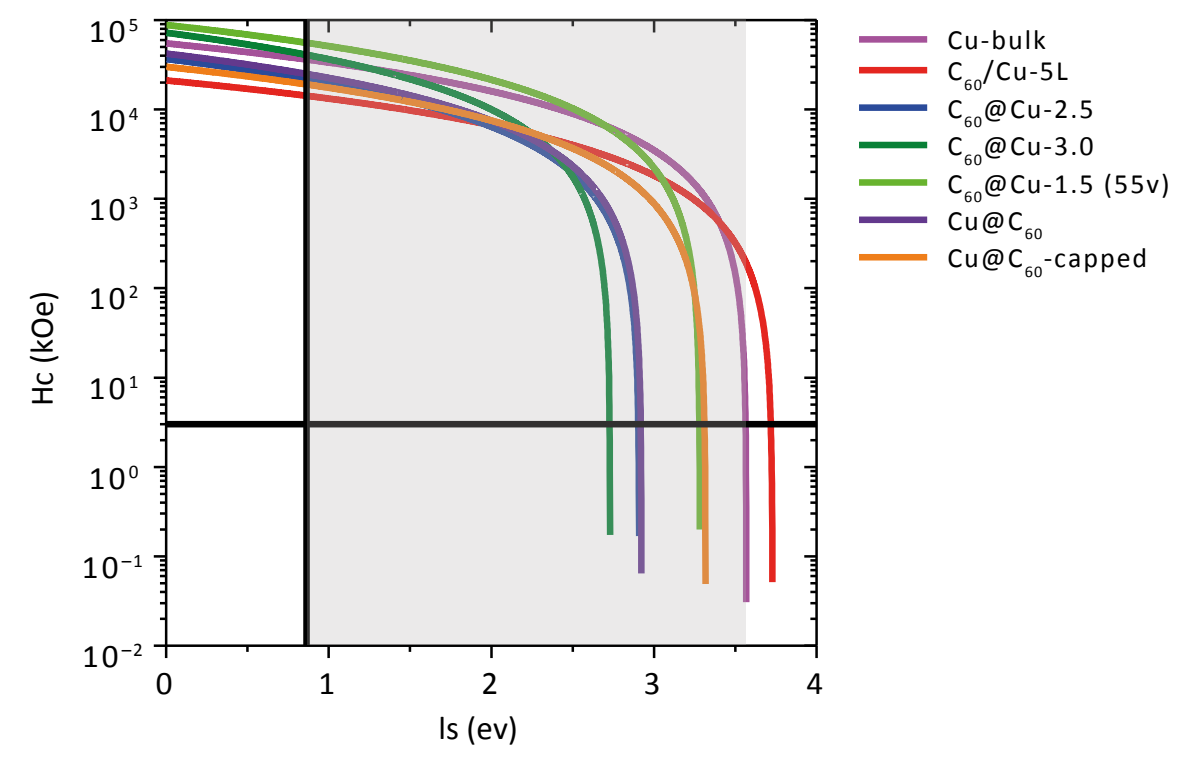

